\title{
Enhanced antitumor activity of trastuzumab emtansine (T-DM1) in combination with pertuzumab in a HER2-positive gastric cancer model
}

\author{
YORIKO YAMASHITA-KASHIMA, SEI SHU, NAOKI HARADA and KAORI FUJIMOTO-OUCHI \\ Product Research Department, Kamakura Research Laboratories, \\ Chugai Pharmaceutical Co., Ltd., Kamakura 247-8530, Japan
}

Received March 27, 2013; Accepted April 29, 2013

DOI: $10.3892 /$ or.2013.2547

\begin{abstract}
Human epidermal growth factor receptor 2 (HER2)targeted therapy by trastuzumab has become increasingly important for treating HER2-positive cancers, and trastuzumab emtansine (T-DM1) is expected to serve as an effective alternative to trastuzumab. Pertuzumab, a HER2 dimerization inhibitor, showed prolonged progression-free survival when used with trastuzumab for HER2-positive breast cancer. In this study, we investigated the effect of combining T-DM1 and pertuzumab on xenografted gastric tumors. T-DM1 as a single agent showed significant antitumor activity in all the three HER2-high expression tumor models tested (NCI-N87, $\mathrm{SCH}$ and 4-1ST) but was ineffective against two HER2-low expression tumors (SNU-16 and MKN-28). Using the T-DM1sensitive NCI-N87 model, the combination efficacy of T-DM1 and pertuzumab was elucidated. The combination induced significant tumor regression, whereas T-DM1 or pertuzumab alone did not. In cultured NCI-N87 cells stimulated with epidermal growth factor (EGF) or heregulin- $\alpha$, concomitant treatment of T-DM1 and pertuzumab significantly inhibited proliferation and increased caspase 3/7 activity compared to either agent alone. Only the combination significantly inhibited the phosphorylation of EGFR or HER3, and its downstream factor AKT. Suppressed HER3 phosphorylation by the combination was also seen in the NCI-N87 xenografted tumors. Compared to single agent treatments, the combination treatment significantly enhanced antibody-dependent cellular cytotoxicity (ADCC) against NCI-N87 cells. These findings suggest that T-DM1 in combination with pertuzumab shows significant antitumor activity by increasing AKT signal inhibition and ADCC in HER2-positive gastric cancers.
\end{abstract}

Correspondence to: Dr Naoki Harada, Product Research Department, Kamakura Research Laboratories, Chugai Pharmaceutical Co., Ltd., 200 Kajiwara, Kamakura 247-8530, Japan

E-mail: haradanok@chugai-pharm.co.jp

Key words: trastuzumab emtansine, T-DM1, pertuzumab, combination, HER2, gastric cancer

\section{Introduction}

For advanced gastric cancers, fluoropyrimidine- and platinumbased combination chemotherapy is currently the most widely accepted treatment. However, its benefit has not been translated into higher overall survival rates. Therefore, there is a need for more effective therapies for gastric cancers.

Overexpression of HER family proteins is often associated with tumor malignancy. Also in gastric cancers, EGFR, HER2, and HER 3 overexpression has been identified and was shown to be associated with prognosis (1-5). Hence, inhibiting the HER family signal transduction is likely to contribute to improved gastric cancer therapies. Indeed, the ToGA trial (6) demonstrated that chemotherapeutic regimens including trastuzumab $\left(\right.$ Herceptin $^{\circledR}$ ), a humanized anti-HER2 antibody, significantly prolonged overall survival of HER2-overexpressing metastatic gastric cancer patients compared to comparison regimens not including trastuzumab.

Trastuzumab emtansine (T-DM1) is a HER2-targeted antibody-drug conjugate (ADC), composed of microtubule polymerization inhibitor DM1 (a derivative of maytansine) linked to trastuzumab. T-DM1 is designed to deliver DM1 into HER2-overexpressing tumor cells. Namely, T-DM1 binds to HER 2 on tumor cells, followed by internalization and degradation in lysosomes. As a consequence, active DM1 is liberated intracellularly from T-DM1 (7-9) and inhibits microtubule assembly causing cell apoptosis/death $(10,11)$. In addition, T-DM1 has been shown to retain the mechanism of action of trastuzumab as an antibody, including antibody-dependent cellular cytotoxicity (ADCC), inhibition of cell signaling through the phosphatidylinositol 3-kinase (PI3K)/AKT pathway, and inhibition of HER2 shedding $(12,13)$. To investigate the clinical effectiveness of T-DM1, two phase III trials, EMILIA (evaluating T-DM1 compared with lapatinib plus capecitabine for patients with HER2-positive metastatic breast cancer previously treated with trastuzumab and a taxane) and MARIANNE (evaluating T-DM1 plus placebo vs. T-DM1 plus pertuzumab vs. trastuzumab plus a taxane for patients with previously untreated HER2-positive metastatic breast cancer) (14) are under way.

Pertuzumab is a humanized anti-HER 2 antibody that exerts antitumor activity in a different manner from trastuzumab. 
Whereas trastuzumab binds to domain IV of the HER2 extracellular domain (ECD), pertuzumab binds to domain II of the HER2 ECD, the region responsible for dimer formation. Thus, pertuzumab inhibits the dimerization of HER2 with other HER family proteins such as EGFR or HER3, which results in prevention of ligand-dependent HER 2 signaling (15). Recently, the efficacy of pertuzumab in HER2-positive metastatic breast cancer was demonstrated in a phase III trial (CLEOPATRA, evaluating trastuzumab plus docetaxel vs. trastuzumab plus docetaxel plus pertuzumab), which showed significantly prolonged progression-free survival (PFS) (16).

Thus, therapies containing anti-HER2 antibodies are expected to be of great clinical significance. In a previous study, we showed an enhanced antitumor activity of trastuzumab in combination with pertuzumab and described its mechanism of action in a HER2-positive gastric cancer model (17). In this context, we believe that the combination of T-DM1 with pertuzumab could be a new potential therapy for advanced gastric cancer. Therefore, in the present study, we investigated the combination effects of T-DM1 and pertuzumab on antitumor activities in vitro and in vivo using HER2-positive human gastric cancers.

\section{Materials and methods}

Test agents. T-DM1 and pertuzumab were provided by F. Hoffmann-La Roche (Basel, Switzerland). Both antibodies were diluted with saline or culture medium in in vivo or in vitro experiments, respectively. Human immunoglobulin G (IgG) was purchased from MP Biomedicals, Inc. (Aurora, OH, USA) and reconstituted with water and diluted with saline.

Animals. Five-week-old male BALB-nu/nu mice (CAnN. Cg-Foxn1<nu $>/$ CrlCrlj nu/nu) were obtained from Charles River Laboratories Japan (Yokohama, Japan). All animals were acclimatized for 1 week prior to the study. The health of the mice was monitored daily. Chlorinated water and irradiated food were provided ad libitum, and the animals were maintained under a controlled light-dark cycle (12 h-12 h). All animal experiments were conducted in accordance with the Institutional Animal Care and Use Committee.

Cell lines and culture. Five human gastric cancer cell lines were used in the present study: NCI-N87, SNU-16, SCH, MKN-28 and 4-1ST. NCI-N87 and SNU-16 cells were purchased from ATCC. SCH cells were purchased from Japan Health Science Foundation (Osaka, Japan). MKN-28 cells were purchased from Immuno-Biological Laboratories Co., Ltd. (Fujioka, Japan). NCI-N87, SNU-16, SCH and MKN-28 were maintained in RPMI-1640 (Sigma-Aldrich, St. Louis, MO, USA), supplemented with $10 \% \mathrm{FBS}$ at $37^{\circ} \mathrm{C}$ under $5 \% \mathrm{CO}_{2}$. 4-1ST cells were purchased from the Central Institute for Experimental Animals (Yokohama, Japan) and maintained in BALB-nu/nu mice by subcutaneous (s.c.) inoculation of pieces of tumor. CD16(158V)/ NK-92 cells were constructed as previously described (17) and were maintained in MEM $\alpha$ (Wako Pure Chemical Industries, Osaka, Japan) supplemented with $12.5 \%$ FBS, $12.5 \%$ horse serum, $0.02 \mathrm{mM}$ folic acid, $0.1 \mathrm{mM}$ 2-mercaptoethanol, $0.2 \mathrm{mM}$ inositol, $0.5 \mathrm{mg} / \mathrm{ml} \mathrm{G} 418$, and $20 \mathrm{ng} / \mathrm{ml}$ recombinant human IL-2 at $37^{\circ} \mathrm{C}$ under $5 \% \mathrm{CO}_{2}$.
Immunohistochemistry and fluorescent in situ hybridization. As previously described, HER2 protein expression and HER2 gene amplification in tumors were examined by immunohistochemistry (IHC) using HercepTest ${ }^{\mathrm{TM}}$ (Dako Japan, Tokyo, Japan) and fluorescent in situ hybridization (FISH) using Path Vysion ${ }^{\circledR}$ (Abbott Japan, Tokyo, Japan), respectively (18).

In vivo tumor growth inhibition studies. Each mouse was inoculated subcutaneously in the right flank with either $5 \times 10^{6}$ cells of human gastric cancer cell lines NCI-N87, SCH, SNU-16 or MKN-28, or with an $\sim 8-\mathrm{mm}^{3}$ piece of 4-1ST tumor tissue. Several weeks after tumor inoculation, mice were randomly allocated to control and treatment groups.

The administration of anticancer agents was started when the tumor volumes reached $\sim 150-350 \mathrm{~mm}^{3}$. T-DM1 was administered intravenously once every 3 weeks and pertuzumab was administered intraperitoneally once a week for 6 weeks. To evaluate the antitumor activity of the test agents, tumor volume was measured twice a week and the percentage of tumor growth inhibition (TGI\%) was calculated as previously described (19).

In vitro anti-proliferation assays. NCI-N87 cells were seeded on 96-well plates at $1 \times 10^{4}$ cells/well and pre-cultured for $24 \mathrm{~h}$. Then the cells were starved in serum-free RPMI-1640 for $24 \mathrm{~h}$ and treated with pertuzumab, T-DM1, or both. Following $30 \mathrm{~min}$ of incubation, cells were exposed to $100 \mathrm{ng} / \mathrm{ml}$ of EGF or heregulin (HRG)- $\alpha$ and incubated for 2 days. Cells were fixed with $10 \%$ formalin neutral buffer solution. Crystal violet staining and extraction were performed as previously described (18) and absorbance was measured at $595 \mathrm{~nm}$. Cells pre-cultured for $24 \mathrm{~h}$ were also detected by crystal violet staining as the absorbance of the pre-cultured well. The percentage of cell proliferation (\% proliferation) was calculated as follows: \% proliferation $=$ (absorbance of treatment well - absorbance of pre-cultured well)/(absorbance of unstimulated well - absorbance of pre-cultured well) x100.

Apoptosis assay. NCI-N87 cells were seeded on 96-well plates at $1 \times 10^{4}$ cells/well and cultured in $0.1 \%$ FBS medium with $100 \mathrm{ng} / \mathrm{ml}$ of EGF or HRG- $\alpha$ added for $24 \mathrm{~h}$. After that, cells were treated with pertuzumab, T-DM1, or both. After $24 \mathrm{~h}$ of treatment, Caspase-Glo ${ }^{\circledR}$ 3/7 Assay (Promega, Madison, WI, USA) was used to measure caspase $3 / 7$ activity, which was calculated as follows: caspase $3 / 7$ activity $=$ (luminescent unit of treatment well - luminescent unit of blank well)/(mean luminescent unit of control well).

Western blotting. NCI-N87 cells were seeded in $0.1 \%$ FBS RPMI-1640 for $24 \mathrm{~h}$ and then treated with $\mathrm{IgG}$ as the control or with $40 \mu \mathrm{g} / \mathrm{ml}$ pertuzumab, $5 \mu \mathrm{g} / \mathrm{ml}$ T-DM1, or both. Following incubation for $1 \mathrm{~h}$, cells were exposed to EGF or HRG- $\alpha$ for 1 or $5 \mathrm{~min}$, respectively. Cells were washed with ice-cold PBS and then lysed with Cell Lysis Buffer (Cell Signaling Technology, Beverly, MA, USA) with $10 \mathrm{mM} \mathrm{NaF}$, $1 \mu \mathrm{g} / \mathrm{ml}$ aprotinin, and $1 \mathrm{mM}$ PMSF. After centrifugation $\left(4^{\circ} \mathrm{C}\right.$, $14,000 \times \mathrm{g}, 5 \mathrm{~min}$ ), the resultant supernatants were used for the western blot assays.

To prepare in vivo samples, tumor samples were taken on day 5 (4 days after treatment) and immediately frozen in liquid 


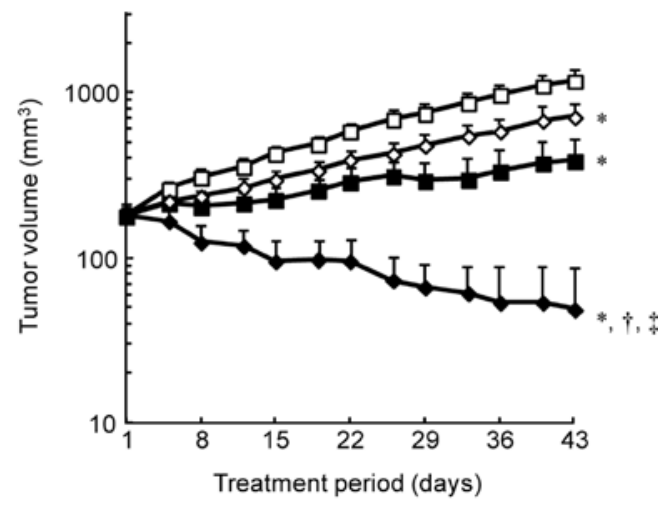

Figure 1. In vivo efficacy of T-DM1 in combination with pertuzumab. Mice bearing NCI-N87 tumors were randomly divided into 4 groups ( $\mathrm{n}=6$ /group) and treated with $40 \mathrm{mg} / \mathrm{kg}$ of HuIgG as a control (open square), $5 \mathrm{mg} / \mathrm{kg}$ of T-DM1 (filled square), $40 \mathrm{mg} / \mathrm{kg}$ of pertuzumab (open rhombus) or $5 \mathrm{mg} / \mathrm{kg}$ of T-DM1 and $40 \mathrm{mg} / \mathrm{kg}$ of pertuzumab (filled rhombus). Data points are mean \pm SD of the tumor volume $\left(\mathrm{mm}^{3}\right)$. Statistically significant differences are shown as ${ }^{*} \mathrm{P}<0.05$ vs. the control group; ${ }^{+} \mathrm{P}<0.05$ vs. the T-DM1 group; ${ }^{\ddagger} \mathrm{P}<0.05$ vs. the pertuzumab group using the Wilcoxon test.

nitrogen and stored at $-80^{\circ} \mathrm{C}$. Tumor samples were homogenized in Cell Lysis Buffer, and centrifuged $\left(4^{\circ} \mathrm{C}, 10,000 \mathrm{x} \mathrm{g}, 20 \mathrm{~min}\right)$. The resultant supernatants were used for the western blot assays.

The protein concentration of the supernatant was quantified using the DC protein assay kit (Bio-Rad Laboratories, Hercules, CA, USA). The supernatants were denatured for $5 \mathrm{~min}$ at $95^{\circ} \mathrm{C}$, electrophoresed on SDS-PAGE and transferred to a polyvinylidene difluoride membrane (Millipore, Bedford, MA, USA). The membrane was blocked in SuperBlock ${ }^{\circledR}$ T20 (TBS) Blocking Buffer (Thermo Scientific, Waltham, MA, USA) and probed with each antibody against HER3 (sc-285), (Santa Cruz Biotechnology, Santa Cruz, CA, USA), HER2 (\#2248), EGFR (\#2963), pEGFR (Tyr1068) (\#3777), pHER2 (Tyr1248) (\#2247), pHER3 (Tyr1289) (\#4791), AKT (\#9272), pAkt (Ser473) (\#9271), ERK1/2 (\#9102), pERK1/2 (Thr202/ Tyr204) (\#9106) (Cell Signaling Technology), and actin (A2228) (Sigma-Aldrich) as primary antibodies. These proteins were detected by horseradish peroxidase-conjugated secondary antibodies. The bands were visualized using Chemi-Lumi One Super (Nacalai Tesque, Kyoto, Japan).

ADCC assays. We used Real-time Cell Analyzer (xCELLigence, Roche Diagnostics, Tokyo, Japan) to monitor ADCC activity in real-time. The system detects electrical impedance on the bottom of the tissue culture E-Plates, which contain interdigitated electrodes as the Cell Index. NCI-N87 cells were seeded on E-plates at $5 \times 10^{3}$ cells/well and pre-cultured for $24 \mathrm{~h} . \mathrm{CD} 16(158 \mathrm{~V}) / \mathrm{NK}-92$ cells were added as effector cells at a target ratio of 1:1. Cells were treated with pertuzumab, T-DM1, or both concurrently. The Cell Index was measured every $5 \mathrm{~min}$ for the first $4 \mathrm{~h}$ and every $10 \mathrm{~min}$ thereafter. The Normalized Cell Index was calculated as follows: (Cell Index at each point $) /($ Cell Index at the point when pertuzumab and T-DM1 treatment started).

Statistical analysis. The Wilcoxon test was used to detect differences in tumor volume for in vivo experiments and Student's t-test was used for in vitro experiments, with $\mathrm{P}<0.05$ considered statistically significant. Statistical analyses were carried out using the SAS preclinical package (SAS Institute, Tokyo, Japan).

\section{Results}

Antitumor activity of T-DMI as a single agent in human gastric cancer mouse xenograft models. Antitumor activity of T-DM1 was evaluated in 5 gastric tumor models with various HER2 levels. NCI-N87, 4-1ST and SCH xenografted tumors exhibited high HER2 expression with IHC scores of $3+$ or $2+$ and a FISH HER2/CEP17 ratio >2.0. SNU-16 and MKN-28 showed low HER2 expression with IHC scores of $1+$ or 0 and a $<2.0$ FISH ratio. On day 22 (21 days after treatment), T-DM1 $(20 \mathrm{mg} / \mathrm{kg}$ ) showed significant antitumor activity in the xenograft models with high HER 2 expression and decreasing tumor volumes. However, T-DM1 did not show significant efficacy for tumors with low HER2 expression (Table I).

Antitumor activity of T-DM1 in combination with pertuzumab in a HER2-positive gastric cancer mouse xenograft model. We assessed the combined efficacy of T-DM1 with pertuzumab using an NCI-N87 xenograft model which expressed high levels of HER 2 and was sensitive to T-DM1 treatment. Compared to each antibody alone, T-DM1 in combination with pertuzumab showed significantly enhanced antitumor activity (Fig. 1).

Combination effect of T-DMI and pertuzumab on proliferation and apoptosis in a HER2-positive human gastric cancer cell line. To investigate the mechanism of action of T-DM1 in combination with pertuzumab, we examined the growth inhibitory effect of T-DM1 and pertuzumab in NCI-N87 cells. These cells have been previously shown to be positive for both HER2 and EGFR and also express low levels of HER3 (17). Pertuzumab completely blocked EGF-stimulated cell proliferation, and T-DM1 stopped cell growth at the initial cell number. In the presence of EGF, T-DM1 combined with pertuzumab significantly enhanced the inhibition of cell proliferation compared to T-DM1 alone (Fig. 2a). We also examined the apoptotic activity of these agents by measuring caspase $3 / 7$ activity. Treatment with both pertuzumab and T-DM1 significantly induced caspase 3/7 activity compared to treatment with either single agent, indicating that pertuzumab in combination with T-DM1 significantly enhanced the apoptosis-inducing activity of T-DM1 (Fig. 2b).

Then, we evaluated the growth inhibition and apoptotic activity induced by T-DM1 in combination with pertuzumab in NCI-N87 cells when stimulated with HRG- $\alpha$. Similar to the stimulation with EGF, combining pertuzumab and T-DM1 significantly enhanced the anti-proliferative activity in HRG- $\alpha$-stimulated cells. Likewise, the increased caspase 3/7 activity seen with pertuzumab and T-DM1 as single agents was further enhanced by their combination under HRG- $\alpha$ (Fig. 2c and d). These results suggest that T-DM1 in combination with pertuzumab has the potential to suppress ligand-dependent cell growth by inducing apoptosis.

Effect of T-DM1 in combination with pertuzumab on HER2 signal transduction. To examine whether pertuzumab and 
Table I. Antitumor activity of T-DM1 as a single agent in human gastric cancer mouse xenograft models.

\begin{tabular}{|c|c|c|c|c|c|c|}
\hline \multirow[b]{2}{*}{ Tumor name } & \multirow{2}{*}{$\begin{array}{l}\text { HER2 } \\
\text { IHC score }\end{array}$} & \multirow{2}{*}{$\begin{array}{c}\text { HER2/CEP17 } \\
\text { ratio (FISH) }\end{array}$} & \multicolumn{3}{|c|}{ Tumor volume $\left(\mathrm{mm}^{3}\right)$} & \multirow[b]{2}{*}{ TGI\% } \\
\hline & & & Treatment & Day 1 & Day 22 & \\
\hline NCI-N87 & $3+$ & $8.4^{\mathrm{b}}$ & $\begin{array}{l}\text { Control } \\
\text { T-DM1 }\end{array}$ & $\begin{array}{l}174 \pm 25 \\
174 \pm 24\end{array}$ & $\begin{array}{c}667 \pm 152 \\
11 \pm 9^{\mathrm{a}}\end{array}$ & 133 \\
\hline $4-1 \mathrm{ST}$ & $3+$ & 16.5 & $\begin{array}{l}\text { Control } \\
\text { T-DM1 }\end{array}$ & $\begin{array}{l}283 \pm 75 \\
283 \pm 72\end{array}$ & $\begin{array}{r}1320 \pm 396 \\
12 \pm 18^{\mathrm{a}}\end{array}$ & 126 \\
\hline $\mathrm{SCH}$ & $2+$ & 2.1 & $\begin{array}{l}\text { Control } \\
\text { T-DM1 }\end{array}$ & $\begin{array}{l}240 \pm 51 \\
241 \pm 63\end{array}$ & $\begin{array}{r}1565 \pm 223 \\
121 \pm 25^{\mathrm{a}}\end{array}$ & 109 \\
\hline SNU-16 & $1+$ & $1.4^{\mathrm{b}}$ & $\begin{array}{l}\text { Control } \\
\text { T-DM1 }\end{array}$ & $\begin{array}{l}185 \pm 23 \\
184 \pm 16\end{array}$ & $\begin{array}{l}485 \pm 116 \\
369 \pm 47\end{array}$ & 39 \\
\hline MKN-28 & 0 & $1.0^{\mathrm{b}}$ & $\begin{array}{l}\text { Control } \\
\text { T-DM1 }\end{array}$ & $\begin{array}{l}271 \pm 50 \\
269 \pm 52\end{array}$ & $\begin{array}{l}1662 \pm 286 \\
1290 \pm 507\end{array}$ & 27 \\
\hline
\end{tabular}

$\mathrm{n}=5-6$, mean $\pm \mathrm{SD}$; ${ }^{\mathrm{P}}<0.05$ by the Wilcoxon test; Day 1, first day of treatment; Day 22, 21 days after starting treatment; TGI\%, $\%$ of tumor growth inhibition; ${ }^{\mathrm{b}}$ citation data from (19).
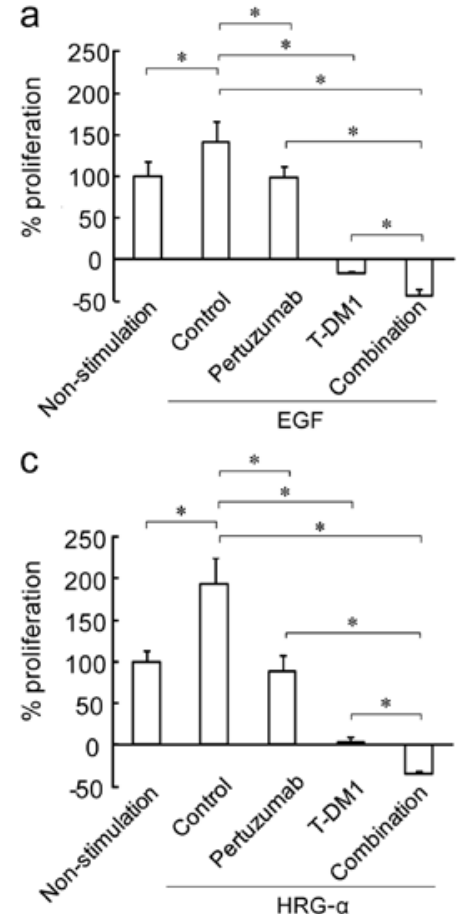

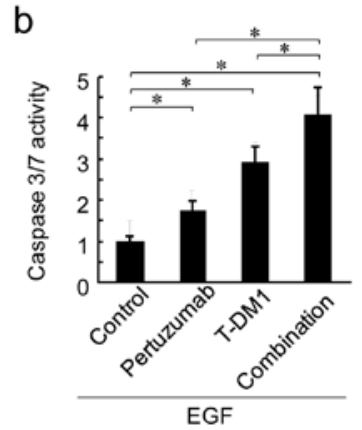

d



Figure 2. In vitro efficacy of T-DM1 combined with pertuzumab on cell proliferation and apoptosis. (a and c) Inhibition of cell growth induced by pertuzumab $(40 \mu \mathrm{g} / \mathrm{ml})$, T-DM1 $(5 \mu \mathrm{g} / \mathrm{ml})$, or both in the presence of EGF (a) or HRG- $\alpha$ (c) was examined in NCI-N87 cells. Control cells were treated with human IgG. Cell proliferation was examined 2 days after treatment. Data points are mean $\pm \mathrm{SD}(\mathrm{n}=5)$. (b and d) Caspase 3/7 activity in cells treated with pertuzumab and trastuzumab combination was measured in the presence of EGF (b) or HRG- $\alpha$ (d). Caspase 3/7 activity in NCI-N87 cells was measured $24 \mathrm{~h}$ after treatment with pertuzumab $(20 \mu \mathrm{g} / \mathrm{ml}), \mathrm{T}-\mathrm{DM} 1(10 \mu \mathrm{g} / \mathrm{ml})$, or both. Data points are mean $\pm \mathrm{SD}(\mathrm{n}=5)$. Statistically significant differences are shown as ${ }^{*} \mathrm{P}<0.05$ using the $\mathrm{t}$-test.

T-DM1 could inhibit HER2 signal transduction, we examined the phosphorylation status of the molecules in HER2-EGFR or HER2-HER3 signaling pathways. Under EGF stimulation, only T-DM1 combined with pertuzumab strongly suppressed phosphor(p)-EGFR, pERK and pAKT (Fig. 3a). Under HRG- $\alpha$ stimulation, pertuzumab alone strongly inhibited pHER3 and, in combination with T-DM1, almost completely suppressed
pHER3 and downstream pAKT. T-DM1 in combination with pertuzumab further reduced pERK in comparison with pertuzumab or T-DM1 alone (Fig. 3b).

To confirm whether the HER family signaling pathway was also inhibited by this therapy in xenografted tumors, tumor specimens collected 4 days after treatment were tested for the phosphorylation level of HER family receptors: pHER2, 
a

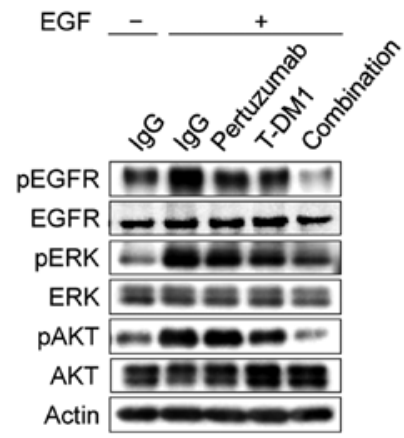

b



C

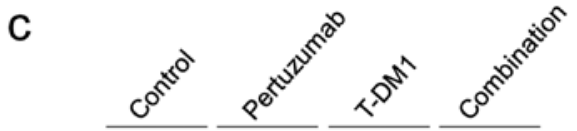

PHER3 -

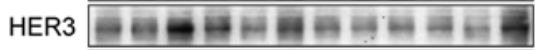

PHER2

HER2 -Evereveren

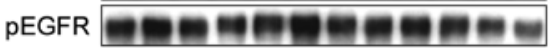

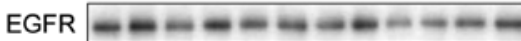

Actin

Figure 3. Effect of T-DM1 in combination with pertuzumab on the signal transduction mediated by HER family proteins. NCI-N87 cells were treated with pertuzumab $(40 \mu \mathrm{g} / \mathrm{ml})$, T-DM1 $(5 \mu \mathrm{g} / \mathrm{ml})$ or both for $1 \mathrm{~h}$ followed by $100 \mathrm{ng} / \mathrm{ml}$ of EGF stimulation for $1 \mathrm{~min}$ (a) or $100 \mathrm{ng} / \mathrm{ml}$ of HRG- $\alpha$ stimulation for $5 \mathrm{~min}$ (b). The phosphorylation levels of HER family proteins and their downstream factors in the cell lysates were detected by western blotting. (c) Tumor samples were homogenized in lysis buffer 4 days after treatment with pertuzumab $(40 \mathrm{mg} / \mathrm{kg})$, T-DM1 $(5 \mathrm{mg} / \mathrm{kg})$, or both. The phosphorylation levels of HER family proteins in the cell lysates were detected by western blotting.

pEGFR and pHER3. pHER2 and pEGFR were slightly reduced whereas pHER3 was clearly reduced by the combination of T-DM1 with pertuzumab compared to either T-DM1 or pertuzumab alone (Fig. 3c).

ADCC activity of T-DM1 in combination with pertuzumab. One way to interpret the different antitumor potency observed in vitro and in vivo is to consider an immune effector-mediated activity, such as ADCC. Hence, we examined whether T-DM1-induced ADCC activity in NCI-N87 cells was further enhanced by pertuzumab, using a real-time cell analyzer. In the wells without effector cells, the addition of pertuzumab and T-DM1 did not reduce the Normalized Cell Index (Fig. 4a). With effector cells, however, the Normalized Cell Index was significantly reduced by the addition of each single agent.

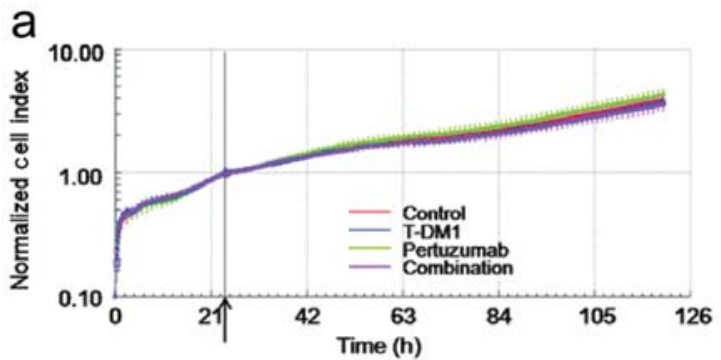

b

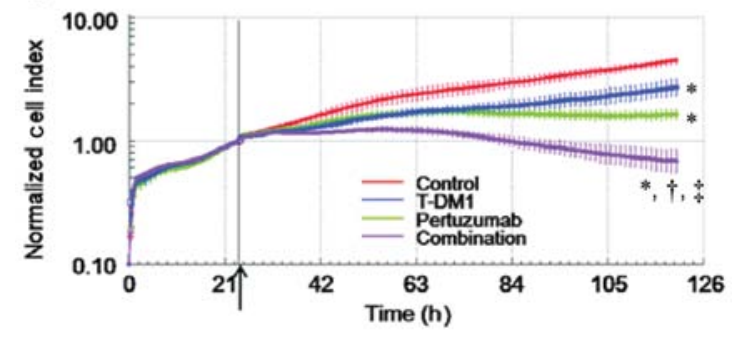

Figure 4. Effect of T-DM1 in combination with pertuzumab on ADCC activity. (a and b) ADCC activity was examined using RTCA (a) without or (b) with NK cells. NCI-N87 cells were treated with T-DM1 (1 ng/ml), pertuzumab $(10 \mathrm{ng} / \mathrm{ml})$, or both. Cell indices were normalized at the treatment point (at the point of arrows). Red, Control (HuIgG-treatment) group; blue, T-DM1 group; green, Pertuzumab group; and magenta, Combination group. Data points are mean $\pm \mathrm{SD}(\mathrm{n}=3)$. Statistically significant differences are shown as ${ }^{*} \mathrm{P}<0.05$ vs. the control group; ${ }^{\dagger} \mathrm{P}<0.05$ vs. the T-DM1 group; and ${ }^{\ddagger} \mathrm{P}<0.05$ vs. the pertuzumab group using the t-test.

Furthermore, T-DM1 in combination with pertuzumab significantly enhanced the reduction of the Normalized Cell Index induced by either T-DM1 or pertuzumab (Fig. 4b), indicating that the combination treatment of T-DM1 and pertuzumab enhanced ADCC activity in vitro.

\section{Discussion}

First, we clarified whether the relationship between HER2 expression level and T-DM1 efficacy in a gastric cancer model was comparable to that of trastuzumab. In breast cancer cells, it is known that T-DM1 shows great antitumor potency to cancer cells with high HER2 expression but less efficacy to those with low HER2 level (8). Our study using gastric cancer xenograft models similarly showed that T-DM1 demonstrated significant antitumor activity only in cancers in which HER2 status was positive (the IHC score was $3+$ or $2+$, and the FISH ratio was $>2.0$ ), suggesting that, as with trastuzumab, efficacy of T-DM1 is associated with HER2 status. On the other hand, antitumor potency of T-DM1 for NCI-N87 (TGI\% was 133\%) was much higher than that of trastuzumab administered at the maximum effective dose in a previous study (TGI\% was 89\%) (18). Even in SCH, in which trastuzumab did not show significant antitumor activity, T-DM1 showed clear antitumor efficacy (TGI was 109\%). Also T-DM1 was efficacious to trastuzumab-insensitive xenograft tumors which expressed HER2 (8). These results confirmed that T-DM1 has more potent antitumor activity than trastuzumab. Further research both in clinical and preclinical studies, however, is required to examine the efficacy of T-DM1 for tumors expressing low levels of HER2, or that are trastuzumab-refractory. 
There are some clinical studies indicating that the combination of pertuzumab and trastuzumab was active and well tolerated in patients with metastatic HER2-positive breast cancer (20). In addition, the CLEOPATRA trial showed that pertuzumab significantly improved PFS of breast cancer patients in combination with trastuzumab plus docetaxel (16) and the combination was thus approved by the FDA. T-DM1 was reported to have significantly improved the PFS of the combination of trastuzumab and docetaxel (21). In addition, the potency of T-DM1 and pertuzumab in combination as a first line treatment in HER2-positive metastatic breast cancers is currently being investigated in the MARIANNE trial. Also, in the ToGA trial investigating gastric cancer, patients with HER2-positive gastric cancer who received trastuzumab in addition to chemotherapy achieved a significant improvement in overall survival compared to treatment with chemotherapy alone (6). From these lines of clinical evidence, next, we studied the combined efficacy of T-DM1 and pertuzumab in HER2-positive gastric cancers. In our in vivo study, the combination treatment of T-DM1 with pertuzumab showed significantly stronger antitumor activity compared to either single agent in the HER2-positive NCI-N87 model, similarly to trastuzumab in combination with pertuzumab (17). T-DM1 in combination with pertuzumab may show strong efficacy in HER2-positive gastric cancer.

Our study demonstrated that the combination of two different anti-HER2 antibodies with different HER2-binding sites enhanced the inhibition of HER2 signal transduction in cultured NCI-N87 cells. Under EGF stimulation, the combination strongly reduced the phosphorylation of EGFR and its downstream factors, ERK and AKT compared to either single agent. Similar results were shown in our previous study in which the combination of trastuzumab and pertuzumab suppressed pEGFR and pHER2 and their downstream pERK and pAKT by inhibiting the EGFR-HER2 heterodimerization more strongly than either single agent (17). These results suggest that T-DM1 in combination with pertuzumab also suppressed heterodimer formation of EGFR-HER2.

In the NCI-N87 cells stimulated with HRG- $\alpha$, concomitant treatment of T-DM1 and pertuzumab almost completely reduced the phosphorylation of HER3 and AKT, and more potently inhibited cell growth and increased apoptotic cells. Furthermore, in the xenografted NCI-N87 tumors, western blot analysis revealed that the combination suppressed the phosphorylation of HER3 not but EGFR. Considering the studies that HER2-HER3 heterodimer is the most active signal transducer among HER family complexes $(22,23)$, it is possible that disruption of the signal of HER3 rather than EGFR may play an important role in the antitumor efficacy of the combination treatment, even though HER3 expression levels were low in NCI-N87 tumors. Although further study is required to elucidate the mechanism of action, suppression of AKT signal followed by apoptosis induction in tumor may contribute to the enhanced antitumor efficacy by pertuzumab combined with T-DM1.

T-DM1 retains the ADCC that is reported as a key mechanism of trastuzumab (24), and pertuzumab also had ADCC $(25,26)$. The results of the present study, which are consistent with the enhanced ADCC activity of pertuzumab combined with trastuzumab in our previous study (17), demon- strate that in the combination treatment using T-DM1 with pertuzumab the ADCC activity was significantly enhanced compared to either single agent alone. On the other hand, a study has shown that the simultaneous binding of trastuzumab and pertuzumab onto target cells did not mediate a synergistic effect on ADCC when they were used at maximum effective doses (25). The authors showed that the level of ADCC of the combination of trastuzumab and pertuzumab was the same as those of the single antibodies, when the final total antibody concentration was identical. The discrepancy may arise from the difference in Materials and methods; that is, we used a real-time cell analyzer to measure ADCC and the sum of the concentration of each antibody was used as the combinational concentration. The results of the present study confirm that, in addition to enhanced inhibition of HER 2 signaling, ADCC may be another mechanism of action that explains the increased efficacy of the combination.

In conclusion, T-DM1 in combination with pertuzumab achieved significant antitumor activity by enhancing HER family signal inhibition and ADCC activity in a HER2positive human gastric cancer model. These findings suggest that T-DM1 in combination with pertuzumab is a rational and promising treatment option for HER2-positive gastric cancer.

\section{Acknowledgements}

We would like to thank Kumiko Kondo, Hiromi Sawamura and Masako Takahashi (Product Research department, Chugai) for their excellent technical assistance; Editing Services, Chugai for helpful advice about drafting the manuscript; and Dr Kazushige Mori for helpful discussion and comments regarding the study.

\section{References}

1. Garcia I, Vizoso F, Martin A, et al: Clinical significance of the epidermal growth factor receptor and HER2 receptor in resectable gastric cancer. Ann Surg Oncol 10: 234-241, 2003.

2. Yonemura Y, Ninomiya I, Yamaguchi A, et al: Evaluation of immunoreactivity for erbB-2 protein as a marker of poor short term prognosis in gastric cancer. Cancer Res 51: 1034-1038, 1991.

3. Tanner M, Hollmen M, Junttila TT, et al: Amplification of HER-2 in gastric carcinoma: association with Topoisomerase IIalpha gene amplification, intestinal type, poor prognosis and sensitivity to trastuzumab. Ann Oncol 16: 273-278, 2005.

4. Nicholson RI, Gee JM and Harper ME: EGFR and cancer prognosis. Eur J Cancer 37 (Suppl 4): S9-S15, 2001.

5. Hayashi M, Inokuchi M, Takagi Y, et al: High expression of HER3 is associated with a decreased survival in gastric cancer. Clin Cancer Res 14: 7843-7849, 2008.

6. Bang YJ, Van Cutsem E, Feyereislova A, et al: Trastuzumab in combination with chemotherapy versus chemotherapy alone for treatment of HER2-positive advanced gastric or gastrooesophageal junction cancer (ToGA): a phase 3 , open-label, randomised controlled trial. Lancet 376: 687-697, 2010.

7. Kovtun YV and Goldmacher VS: Cell killing by antibody-drug conjugates. Cancer Lett 255: 232-240, 2007.

8. Lewis Phillips GD, Li G, Dugger DL, et al: Targeting HER2-positive breast cancer with trastuzumab-DM1, an antibody-cytotoxic drug conjugate. Cancer Res 68: 9280-9290, 2008.

9. Erickson HK, Lewis Phillips GD, Leipold DD, et al: The effect of different linkers on target cell catabolism and pharmacokinetics/ pharmacodynamics of trastuzumab maytansinoid conjugates. Mol Cancer Ther 11: 1133-1142, 2012.

10. Oroudjev E, Lopus M, Wilson L, et al: Maytansinoid-antibody conjugates induce mitotic arrest by suppressing microtubule dynamic instability. Mol Cancer Ther 9: 2700-2713, 2010. 
11. Lopus M, Oroudjev E, Wilson L, et al: Maytansine and cellular metabolites of antibody-maytansinoid conjugates strongly suppress microtubule dynamics by binding to microtubules. Mol Cancer Ther 9: 2689-2699, 2010

12. Junttila TT, Li G, Parsons K, Phillips GL and Sliwkowski MX: Trastuzumab-DM1 (T-DM1) retains all the mechanisms of action of trastuzumab and efficiently inhibits growth of lapatinib insensitive breast cancer. Breast Cancer Res Treat 128: 347-356, 2011.

13. Barok M, Tanner M, Koninki K and Isola J: Trastuzumab-DM1 is highly effective in preclinical models of HER2-positive gastric cancer. Cancer Lett 306: 171-179, 2011.

14. LoRusso PM, Weiss D, Guardino E, Girish S and Sliwkowski MX: Trastuzumab emtansine: a unique antibody-drug conjugate in development for human epidermal growth factor receptor 2-positive cancer. Clin Cancer Res 17: 6437-6447, 2011.

15. Franklin MC, Carey KD, Vajos FF, Leahy DJ, de Vos AM and Sliwkowski MX: Insights into ErbB signaling from the structure of the ErbB2-pertuzumab complex. Cancer Cell 5: 317-328, 2004.

16. Baselga J, Cortes J, Kim SB, et al: Pertuzumab plus trastuzumab plus docetaxel for metastatic breast cancer. N Engl J Med 366: 109-119, 2012.

17. Yamashita-Kashima Y, Iijima S, Yorozu K, et al: Pertuzumab in combination with trastuzumab shows significantly enhanced antitumor activity in HER2-positive human gastric cancer xenograft models. Clin Cancer Res 17: 5060-5070, 2011.

18. Fujimoto-Ouchi K, Sekiguchi F, Yasuno H, Moriya Y, Mori K and Tanaka Y: Antitumor activity of trastuzumab in combination with chemotherapy in human gastric cancer xenograft models. Cancer Chemother Pharmacol 59: 795-805, 2007.

19. Fujimoto-Ouchi K, Sekiguchi F and Tanaka Y: Antitumor activity of combinations of anti-HER-2 antibody trastuzumab and oral fluoropyrimidines capecitabine/5'-dFUrd in human breast cancer models. Cancer Chemother Pharmacol 49: 211-216, 2002.
20. Baselga J, Gelmon KA, Verma S, et al: Phase II trial of pertuzumab and trastuzumab in patients with human epidermal growth factor receptor 2-positive metastatic breast cancer that progressed during prior trastuzumab therapy. J Clin Oncol 28: $1138-1144,2010$

21. Hurvitz SA, Dirix L, Kocsis J, Gianni L, Lu MJ, Vinholes J, Song C, Tong B, Chu Y-W and Perez EA: Trastuzumab emtansine (T-DM1) vs trastuzumab plus docetaxel $(\mathrm{H}+\mathrm{T})$ in previously untreated HER2-positive metastatic breast cancer (MBC): primary results of a randomized, multicenter, open-label phase II study (TDM4450g/BO21976). Eur J Cancer 47: S330, 2011. doi: 10.1016/S0959-8049(11)71443-5.

22. Baselga $\mathbf{J}$ and Swain SM: Novel anticancer targets: revisiting ERBB2 and discovering ERBB3. Nat Rev Cancer 9: 463-475, 2009.

23. Olayioye MA, Neve RM, Lane HA and Hynes NE: The ErbB signaling network: receptor heterodimerization in development and cancer. EMBO J 19: 3159-3167, 2000.

24. Spector NL and Blackwell KL: Understanding the mechanisms behind trastuzumab therapy for human epidermal growth factor receptor 2-positive breast cancer. J Clin Oncol 27: 5838-5847, 2009.

25. Scheuer W, Friess T, Burtscher H, Bossenmaier B, Endl J and Hasmann M: Strongly enhanced antitumor activity of trastuzumab and pertuzumab combination treatment on HER2-positive human xenograft tumor models. Cancer Res 69: 9330-9336, 2009.

26. El-Sahwi K, Bellone S, Cocco E, et al: In vitro activity of pertuzumab in combination with trastuzumab in uterine serous papillary adenocarcinoma. Br J Cancer 102: 134-143, 2010. 